\title{
COMPORTAMENTOS SEXUAIS NA INFÂNCIA E ASSOCIAÇÃO COM PROBLEMAS DE COMPORTAMENTO
}

\section{Marina Monzani da Rocha}

Programa de Pós-Graduação em Distúrbios do Desenvolvimento - Universidade Presbiteriana Mackenzie, Brasil.

\section{Camila Teixeira Monteiro}

Universidade Presbiteriana Mackenzie, Brasil.

Ana Lucia Alcântara de Oliveira Ulian

Universidade Federal da Bahia, Brasil.

Edwiges Ferreira de Mattos Silvares

Universidade de São Paulo, Brasil.
RESUMO: Com objetivo de verificar a ocorrência de diferentes comportamentos sexuais na infância, a partir da perspectiva dos pais, e suas relações com a idade da criança, o sexo e a presença de problemas de comportamento, pais de 54 crianças ( 4 a 8 anos), sem histórico de problemas de saúde mental ou de abuso sexual, responderam ao "Inventário de Comportamentos Sexuais da Criança" (CSBI) e o "Inventário dos Comportamentos de Crianças e Adolescentes" (CBCL/4-18). Foram reportados diferentes tipos de comportamentos sexuais com variada frequência. Pais de meninos relataram, em média, mais comportamentos sexuais que os de meninas $(8,04$ x 5,12$)$. Não foi encontrada diferença estatística entre o comportamento sexual de pré-escolares e escolares. Os comportamentos sexuais mais e menos frequentemente apresentados pela amostra foram semelhantes aos de estudos internacionais. Correlação positiva moderada foi estabelecida entre comportamentos sexuais (CSBI) e problemas de comportamento (CBCL). Levantamentos como este devem auxiliar a avaliação de problemas na área e facilitar a orientação aos pais e às crianças sobre a sexualidade infantil.

PALAVRAS-CHAVE: Criança; Comportamento Sexual; Desenvolvimento Infantil; Problema de Comportamento.

\section{SEXUAL BEHAVIOR IN CHILDHOOD AND ASSOCIATION WITH BEHAVIOR PROBLEMS}

ABSTRACT: To verify the occurrence of sexual behavior during childhood, from the perspective of the parents, and its relation with child's age, gender, and the presence of behavioral problems, parents of 54 children ( 4 to 8 years old), with no history of mental health issues or sexual abuse, filled the "Child Sexual Behavior Inventory" (CSBI) and the "Child Behavior Checklist" (CBCL/4-18). A wide variation of the sexual behaviors, with different frequency, was reported. Parents of boys reported, on average, more sexual behaviors than parents of girls ( 8.04 vs. 5.12$)$. No statistical difference was found between the frequency of sexual behaviors in preschoolers and school age children. Moderated positive correlations were found between sexual behaviors (CSBI) and behavioral problems (CBCL). The most and least frequent sexual behaviors presented by our sample were similar to those found in international studies. Surveys like the one conducted with this sample should assist the evaluation of problems in the area, and facilitate guidance for parents and children on child sexuality.

KEY WORDS: Children; Sexual behavior; Child development; Behavior issues. 


\section{INTRODUÇÃO}

Comportamentos sexuais são comuns e frequentemente observáveis em crianças. Muitos desses comportamentos são típicos e apropriados para as diferentes etapas do desenvolvimento, enquanto outros não são. Independente desse caráter, os comportamentos são transitórios e ocorrem dentro de toda trajetória do crescimento humano. A gama desses comportamentos vai desde comportamentos exploratórios movidos pela curiosidade e comportamentos de teste dos laços afetivos eliciados por fatores situacionais, até os abusivos, violentos e intrusivos, que podem estar associados com diversos fatores familiares ou circunstâncias, tais como abuso sexual ou físico e negligência ${ }^{1}$.

O conhecimento do desenvolvimento normal da sexualidade infantil é imprescindível aos pais e profissionais que trabalham com crianças de modo que estes possam identificar quais comportamentos são comuns para uma determinada idade e reconhecer os comportamentos inapropriados e abusivos, que merecem atenção clínica ${ }^{1}$.

Embora as pesquisas sobre sexualidade infantil apresentem limitações, especialmente de ordem metodológica, pois geralmente os dados são colhidos indiretamente, pelos pais e professores, ou de forma retrospectiva, em entrevistas com adultos sobre suas memórias de comportamentos sexuais na infância ${ }^{2}$, diversos estudos com o objetivo de avaliar os tipos de comportamento sexuais comumente apresentados na infância vêm sendo realizados, principalmente nas duas últimas décadas. Sinteticamente falando, a conclusão geral é de que uma grande variedade de comportamentos sexuais, como tocar ou mostrar a terceiros as partes sexuais (privadas), ou falas de cunho sexual, é apresentada por crianças sem histórico de abuso sexual ${ }^{1,3,4-7}$.

Uma cuidadosa revisão da literatura sobre sexualidade infantil, com o objetivo de identificar comportamentos sexuais apresentados desde o nascimento até os 12 anos, reportou aqueles que são mais frequentemente observados nas diferentes etapas do desenvolvimento ${ }^{6}$. Para os recém-nascidos e bebês, os comportamentos sexuais frequentemente apontados foram, por exemplo, o sugar dos dedos das mãos e dos pés dos fetos; a ereção peniana e lubrificação vaginal e as respostas orgásticas em bebês de cinco meses. Já para os pré-escolares, a masturbação, brincadeiras de sexo com os pares, exploração dos próprios órgãos sexuais e dos outros, exibicionismo e voyeurismo estiveram dentre os mais frequentes; e, para as crianças dos seis aos 12 anos, foram ressaltados os jogos sexuais intensificados, com masturbação mútua e individual, fantasias sexuais, beijos, simulação de intercurso, mudanças corporais e constrangimento perante os adultos.

Um estudo com grande amostra populacional $(\mathrm{N}=880)$ foi realizado nos Estados Unidos para avaliar a frequência com que uma variedade de comportamentos sexuais era reportada por pais de crianças de dois a 12 anos sem histórico de abuso sexual, dificuldade física ou mental e encaminhamento para serviços de saúde mental nos últimos seis meses ${ }^{4}$. Utilizando o "Child Sexual Behavior Inventory" (CSBI) ${ }^{4}$, pesquisadores encontraram que "brincar com brinquedos do sexo oposto" e "causar machucados na região anal ou entre as coxas" são comportamentos apresentados por mais que $50 \%$ da amostra. Por outro lado, "colocar a boca nas partes sexuais de outras crianças ou adultos", "pedir a outras pessoas para participarem de atividade sexual consigo", "masturbar-se com objeto" e "inserir ou tentar inserir objetos na vagina ou ânus" são comportamentos raramente encontrados nas crianças destas faixas etárias.

Neste estudo, também foi identificado que crianças mais velhas, de ambos os sexos, apresentaram menos comportamentos sexuais que as mais novas, ou seja, uma variedade de comportamentos sexuais, que parecem ser típicos para crianças pequenas, "desaparecem" com o aumento da idade. Além disso, as crianças que apresentaram mais comportamentos sexuais no CSBI (independentemente da idade) apresentaram também problemas de comportamento no "Inventário dos Comportamentos de Crianças e Adolescentes de 4 a 18 anos" ( $\mathrm{CBCL}^{8}$ ), demonstrando que há relação entre a frequência de comportamentos sexuais e problemas de comportamento, de modo geral ${ }^{4}$.

Em 1998, os mesmos autores procuraram atualizar o trabalho desenvolvido em $1991^{4}$ e encontraram frequências de comportamento sexuais semelhantes ao estudo anterior ${ }^{5}$. Com ênfase na relação entre compor- 
tamentos sexuais e problemas de comportamento, afirmaram que crianças com problemas sexuais têm maior probabilidade de apresentar problemas de internalização, como depressão, ansiedade e isolamento, e de externalização, como agressão, violação de regras e hiperatividade 5 . Tal associação pode ser um indício de que alguns problemas sexuais ocorrem em um contínuo com os problemas comportamentais e que as causas são multifatoriais ${ }^{1}$.

Considerando que diferenças nas atitudes e valores com relação à sexualidade, principalmente com relação à sexualidade infantil, podem interferir na ocorrência dos comportamentos, um estudo semelhante foi elaborado para conhecer os comportamentos sexuais das crianças na Bélgica ${ }^{7}$. Utilizado o CSBI, os autores Schoentjes, Deboutte e Friedrich confirmaram que comportamentos sexuais são frequentes em crianças e que sua ocorrência depende da fase de desenvolvimento e de fatores individuais e familiares. Não foram encontradas diferenças significativas entre o comportamento das crianças belgas e americanas?

Em função da associação existente entre comportamentos sexuais e estresse pós-traumático, além do aumento significativo de comportamentos sexuais em crianças que sofreram abuso sexual, foi realizado um estudo para comparar a frequência de comportamentos sexuais de crianças sem histórico de abuso ou encaminhamento para serviços de saúde-mental, com o de crianças abusadas e de crianças não abusadas encaminhadas para serviços de saúde mental'. Os autores encontraram que as crianças abusadas apresentam, de fato, mais comportamentos sexuais que as dos outros dois grupos, e que as crianças encaminhadas para serviços de saúde mental apresentam mais comportamentos sexuais que as crianças sem histórico de abusou ou encaminhamento. A correlação entre comportamentos sexuais e problemas de comportamento, já apontada em outros estudos ${ }^{4 \cdot 6}$, foi confirmada para essa amostra ${ }^{9}$.

Um estudo recente, envolvendo ampla amostra ( $N=1112$ ), foi realizado a partir do relato de professores, cuidadores e crianças referentes a problemas de comportamento sexuais ${ }^{3}$. Neste, foram analisados os problemas comportamentais internalizantes, externalizantes e sociais de crianças com e sem problemas de comportamen- to sexuais reportados pelos cuidadores. Os resultados obtidos apontaram que os cuidadores indicam maiores índices de problemas de comportamento e de relacionamento social em crianças que apresentam problemas sexuais em comparação com aquelas que não apresentam. As preocupações dos cuidadores raramente foram corroboradas pelos professores e pelas crianças. Dessa forma, os autores sugerem o uso de múltiplos informantes para avaliação de problemas sexuais na infância, especialmente considerando os riscos relacionados com informações equivocadas ou incompletas ${ }^{3}$.

Ainda que o comportamento sexual, como os outros tipos de comportamento, esteja sujeito às influências culturais, em função do que é aceito em cada norma social e do que é exibido na mídia, e as influências dos hábitos familiares (como, por exemplo, nudez em casa e abertura a diálogo sobre questões sexuais), os resultados dos estudos realizados em diferentes sociedades são muito semelhantes ${ }^{4,5,7}$.

Levantamentos como os expostos acima devem funcionar como parâmetro para avaliação de comportamentos sexuais em crianças e problemas nesta área. Esse tipo de avaliação se justifica pela importância de conhecer os comportamentos típicos para poder-se realizar diagnósticos e trabalhos preventivos de problemas de ordem sexual, como abuso sexual e diforia de gênero. Em função da necessidade de se realizar mais pesquisas desta natureza, o presente trabalho pretende avaliar a frequência de comportamentos sexuais observados por pais de crianças, estudar o efeito de idade e o sexo na presença e nos tipos de comportamentos apresentados, além de verificar a relação entre comportamentos sexuais e problemas de comportamento em uma amostra de crianças brasileiras.

\section{METODOLOGIA}

\section{DELINEAMENTO}

Foi realizado um estudo quantitativo, de caráter transversal, com objetivo de descrever os comportamentos observados na amostra e analisar a relação entre as variáveis comportamento sexual, sexo, idade e problemas de comportamento. 


\section{PARTICIPANTES}

Participaram desta pesquisa 54 pais de crianças com idades entre quatro e oito anos (média $=5,78$, desvio-padrão $=1,24)$, sendo 28 meninos e 26 meninas. Destas, 23 estavam na faixa etária pré-escolar $(04$ e 05 anos), enquanto que 31 tinham de seis a oito anos. Não há diferença na distribuição por sexo nas duas faixas etárias $(\chi 2(1)=0,260, p=0,610)$.

Todas as crianças apresentavam desenvolvimento típico e não tinham histórico de abuso sexual. As famílias residiam nos Estados de São Paulo e Bahia e foram recrutadas por conveniência em escolas de educação infantil e clínicas pediátricas.

\section{INSTRUMENTOS}

1) "Inventário do Comportamento Sexual Infantil" - (tradução do Child Sexual Behavior Inventory - CSBI ${ }^{4}$ : inventário destinado a medir a frequência de comportamentos sexuais apresentados recentemente ou nos últimos seis meses por crianças e pré-adolescentes, a partir do relato dos pais. É composto por 35 questões com uma escala que varia de 0 a 3 , sendo: $0=$ nunca, 1 $=$ menos de 1 vez por mês, $2=1-3$ vezes no mês, $3=$ pelo menos 1 vez por semana. $O$ instrumento foi adaptado para o português. ${ }^{11}$

2) "Inventário dos Comportamentos de Crianças e Adolescentes de 4 a 18 anos" (versão brasileira do Child Behavior Checklist - CBCL/4-18 8 ): instrumento de avaliação norte-americano, adaptado para uso no Brasil. ${ }^{10}$ É um inventário que avalia problemas de comportamento de crianças e adolescentes a partir do relato dos pais. São apresentados 113 itens, agrupados em escala de Internalização, escala de Externalização e escala Total de Problemas Emocionais/Comportamentais. A pontuação obtida em cada uma dessas escalas pode atingir a faixa normal, limítrofe e clínica, de acordo com a intensidade e/ou frequência de problemas reportados pelos pais.

\section{PROCEDIMENTO}

O critério de inclusão para o estudo foi a criança estar na faixa etária dos quatro a oito anos, por: a) corresponder a faixa etária examinada no estudo norte-america- no ${ }^{4}$; b) adequar-se à idade mínima do $\mathrm{CBCL} / 4-18$ e c) pelo fato de as crianças com até oito anos não terem ainda entrado na faixa de pré-adolescência, caracterizando assim o grupo como infantil.

Os pesquisadores entraram em contato com os responsáveis pelas crianças, explicaram o objetivo do trabalho e os convidaram a participar do estudo. Aqueles que aceitaram participar assinaram o Termo de Consentimento Livre e Esclarecido (TCLE) e receberam os dois questionários (CSBI e CBCL), com explicação de como deveriam ser preenchidos. Foi explicitada a importância de não deixarem nenhum item em branco. Uma forma para devolução dos questionários foi combinada nos casos em que os mesmos não podiam ser preenchidos no momento do encontro. Foram observados todos os princípios éticos pregados na resolução 466/2012 do Conselho Nacional de Saúde. Ao término da pesquisa, todos os participantes receberam um relatório sintético com os resultados obtidos.

Os dados foram analisados utilizando o software estatístico SPSS 20.0. Para avaliar a frequência dos diversos comportamentos sexuais reportados pelos pais das crianças, a porcentagem de crianças para as quais o item foi assinalado foi calculada em função de sua ocorrência, independentemente de os pais terem assinalado que o comportamento ocorreu menos de uma vez por mês, uma a três vezes no mês, ou pelo menos uma vez por semana. A comparação da frequência dos diferentes itens dentre as crianças escolares e pré-escolares e dentre meninos e meninas foi realizada utilizando o teste quiquadrado. Testes t de Student para amostras independentes foram realizados para verificar diferenças nas médias obtidas em cada um dos grupos. Para todas as análises foi adotado o nível de significância de 95\%.

\section{RESULTADO}

1) Frequência de comportamentos sexuais nas crianças brasileiras

Para avaliar a frequência dos diversos comportamentos sexuais, a porcentagem de crianças para as quais o item foi assinalado foi calculada em função de sua ocorrência, independentemente de os pais terem assinalado 
que o comportamento ocorreu menos de uma vez por mês, uma a três vezes no mês, ou pelo menos uma vez por semana. Assim, as porcentagens apresentadas na Tabela 1 se referem à presença de cada comportamento pelo menos uma vez nos últimos seis meses, independentemente da frequência ou intensidade que ele teve. Os resultados estão divididos por sexo e idade em função das possíveis diferenças associadas a essas variáveis.

Tabela 1. Porcentagem de afirmação dos itens do CSBI

(Continua)

\begin{tabular}{|c|c|c|c|c|c|}
\hline Item & Geral & $\begin{array}{c}0-4 \\
\text { e } 5 \\
\text { anos } \\
N= \\
11\end{array}$ & $\begin{array}{c}o-6 \\
\text { a } 8 \\
\text { anos } \\
\mathrm{N}= \\
17\end{array}$ & $\begin{array}{c}+ \text { - } 4 \\
\text { e } 5 \\
\text { anos } \\
\mathrm{N}= \\
12\end{array}$ & $\begin{array}{c}\text { P - } 6 \\
\text { a } 8 \\
\text { anos } \\
\mathrm{N}= \\
14\end{array}$ \\
\hline $\begin{array}{l}\text { 1. Veste-se como se fosse do } \\
\text { sexo oposto }\end{array}$ & $5,6 \%$ & $9,1 \%$ & $11,8 \%$ & $0,0 \%$ & $0,0 \%$ \\
\hline 2. Deseja ser do sexo oposto & $7,4 \%$ & $9,1 \%$ & $11,8 \%$ & $8,3 \%$ & $0,0 \%$ \\
\hline $\begin{array}{l}\text { 3. Toca as partes sexuais em } \\
\text { público }\end{array}$ & $18,5 \%$ & $18,2 \%$ & $23,5 \%$ & $16,7 \%$ & $14,3 \%$ \\
\hline 4. Masturba-se com as mãos & $14,8 \%$ & $18,2 \%$ & $17,6 \%$ & $16,7 \%$ & $7,1 \%$ \\
\hline $\begin{array}{l}\text { 5. Causa machucados nas } \\
\text { coxas e/ou ânus }\end{array}$ & $0,0 \%$ & $0,0 \%$ & $0,0 \%$ & $0,0 \%$ & $0,0 \%$ \\
\hline $\begin{array}{l}\text { 6. Toca em seios (da mãe ou de } \\
\text { outra mulher) }\end{array}$ & $44,4 \%$ & $54,5 \%$ & $47,1 \%$ & $50,0 \%$ & $28,6 \%$ \\
\hline 7. Masturba-se com objetos & $1,9 \%$ & $0,0 \%$ & $0,0 \%$ & $8,3 \%$ & $0,0 \%$ \\
\hline $\begin{array}{l}\text { 8. Toca as partes sexuais dos } \\
\text { outros }\end{array}$ & $14,8 \%$ & $27,3 \%$ & $17,6 \%$ & $8,3 \%$ & $7,1 \%$ \\
\hline 9. Imita ato sexual & $16,7 \%$ & $18,2 \%$ & $29,4 \%$ & $16,7 \%$ & $0,0 \%$ \\
\hline $\begin{array}{l}\text { 10. Colocar boca em partes se- } \\
\text { xuais }\end{array}$ & $1,9 \%$ & $0,0 \%$ & $0,0 \%$ & $8,3 \%$ & $0,0 \%$ \\
\hline 11. Toca os genitais em casa & $51,9 \%$ & $54,5 \%$ & $64,7 \%$ & $41,7 \%$ & $42,9 \%$ \\
\hline 12. Usa palavras sexuais & $20,4 \%$ & $9,1 \%$ & $47,1 \%$ & $0,0 \%$ & $14,3 \%$ \\
\hline 13. Finge ser do sexo oposto & $11,1 \%$ & $18,2 \%$ & $17,6 \%$ & $8,3 \%$ & $0,0 \%$ \\
\hline 14. Reproduz barulhos sexuais & $9,3 \%$ & $9,1 \%$ & $17,6 \%$ & $8,3 \%$ & $0,0 \%$ \\
\hline $\begin{array}{l}\text { 15. Pede para participar de atos } \\
\text { sexuais }\end{array}$ & $1,9 \%$ & $0,0 \%$ & $5,9 \%$ & $0,0 \%$ & $0,0 \%$ \\
\hline $\begin{array}{l}\text { 16. Esfrega seu corpo em } \\
\text { outras pessoas }\end{array}$ & $18,5 \%$ & $18,2 \%$ & $17,6 \%$ & $16,7 \%$ & $21,4 \%$ \\
\hline $\begin{array}{l}\text { 17. Insere objetos no ânus ou } \\
\text { vagina }\end{array}$ & $0,0 \%$ & $0,0 \%$ & $0,0 \%$ & $0,0 \%$ & $0,0 \%$ \\
\hline $\begin{array}{l}\text { 18. Tenta ver pessoas } \\
\text { despindo-se }\end{array}$ & $29,6 \%$ & $36,4 \%$ & $29,4 \%$ & $33,3 \%$ & $21,4 \%$ \\
\hline $\begin{array}{l}\text { 19. Imita atos sexuais com } \\
\text { bonecos }\end{array}$ & $9,3 \%$ & $9,1 \%$ & $11,8 \%$ & $8,3 \%$ & $7,1 \%$ \\
\hline
\end{tabular}

(Conclusão)

20. Mostra as partes sexuais para adultos

\begin{tabular}{l|l|l|l|l|l}
\hline 21. Olha foto de pessoas nuas & $29,6 \%$ & $9,1 \%$ & $52,9 \%$ & $25,0 \%$ & $21,4 \%$ \\
\hline
\end{tabular}

\begin{tabular}{l|l|l|l|l|l}
\hline 22. Fala sobre atos sexuais & $11,1 \%$ & $0,0 \%$ & $29,4 \%$ & $0,0 \%$ & $7,1 \%$ \\
\hline
\end{tabular} \begin{tabular}{l|l|l|l|l|l}
\hline $\begin{array}{l}\text { 23. Beija adultos que não são } \\
\text { da família }\end{array}$ & $29,6 \%$ & $36,4 \%$ & $41,2 \%$ & $16,7 \%$ & $21,4 \%$ \\
\hline
\end{tabular}

24. Tira a roupa na frente dos outros

\begin{tabular}{|l|l|l|l|l}
$44,4 \%$ & $63,6 \%$ & $41,2 \%$ & $41,7 \%$ & $35,7 \%$
\end{tabular} \begin{tabular}{l|l|l|l|l|l}
\hline $\begin{array}{l}\text { 25. Senta-se expondo as coxas } \\
\text { ou roupas intimas }\end{array}$ & $24,1 \%$ & $18,2 \%$ & $17,6 \%$ & $25,0 \%$ & $35,7 \%$ \\
\hline
\end{tabular}

26. Beija crianças que não são da família

\begin{tabular}{l|l|l|l|l|l|l|}
\hline 27. Fala de maneira sedutora & $29,6 \%$ & $27,3 \%$ & $41,2 \%$ & $33,3 \%$ & $14,3 \%$ \\
\hline
\end{tabular}

\begin{tabular}{l|l|l|l|l|l}
\hline $\begin{array}{l}28 . \text { Tira a roupa de outras } \\
\text { pessoas }\end{array}$ & $7,4 \%$ & $0,0 \%$ & $17,6 \%$ & $8,3 \%$ & $0,0 \%$ \\
\hline
\end{tabular}
pessoas

29. Pede para assistir conteúdo explicito na TV

30. Tenta colocar a língua no beijo

31. Abraça adultos desconhecidos

32. Mostra partes sexuais para outras crianças

33. Menina agressiva, menino passivo

\begin{tabular}{l|l|l|l|l|l|l|l}
\hline 34. Interesse pelo sexo oposto & $46,3 \%$ & $63,6 \%$ & $52,9 \%$ & $33,3 \%$ & $35,7 \%$ \\
\hline
\end{tabular}

\begin{tabular}{|l|l|l|l|l|l}
\hline 35. Brinca com brinquedos do & $46,3 \%$ & $63,6 \%$ & $47,1 \%$ & $50,0 \%$ & $28,6 \%$ \\
\hline
\end{tabular}
sexo oposto

Legenda: $\widehat{\sigma}=$ meninos; $ᄋ=$ meninas

No geral, 13 dos 35 itens foram assinalados para mais de $20 \%$ da amostra, sendo que o item mais assinalado foi " 11 : Toca as partes sexuais (privadas) quando está em casa", com pouco mais que a metade dos pais $(51,9 \%)$ afirmando observar esse comportamento em seus filhos. Os comportamentos presentes nos itens mais frequentemente identificados podem ser considerados comuns para a faixa etária.

Paralelamente, cinco itens foram assinalados por menos que $2 \%$ da amostra, dentre eles dois que não foram apontados por nenhum dos pais: "5. Causa machucados na região anal e nas coxas por excesso de manuseio" e "17. Insere ou tenta inserir objetos na vagina e/ou ânus". Os itens menos assinalados representam comportamentos pouco frequentes para essa amostra e sua presença 
pode ser indício de que a criança que os apresenta está com problemas nessa área. É interessante notar que os pais de seis crianças da amostra responderam que seus filhos não apresentam qualquer um dos comportamentos sexuais.

A comparação dos resultados obtidos pelos quatro grupos (meninos pré-escolares, meninos escolares, meninas pré-escolares e meninas escolares) revelou que os meninos escolares apresentam maior frequência de uso de palavras sexuais (item 12) $(\chi 2(3)=17,717, p=$ $0,008)$ e de falas sobre atos sexuais (item 22) $(\chi 2(3)=$ $8,863, p=0,031)$. Além disso, eles mostram seus órgãos sexuais para adultos com mais frequência que as meninas de sua faixa etária (item 20$)(\chi 2(3)=10,688, p=0,014)$. De fato, a análise da Tabela 1 indica que 52,9\% dos pais de meninos de seis a oito anos reportam que seus filhos mostram seus órgãos sexuais para adultos, enquanto que nenhum dos pais de meninas reportou esse item como presente nos últimos seis meses.

Analisando apenas os dados dos meninos, encontramos maior frequência de escolares utilizando palavras sexuais (item 12) $(\chi 2(1)=4,414, p=0,036)$, olhando fotos de pessoas nuas (item 21) $(\chi 2(1)=5,593$, $p=0,018)$ e falando sobre atos sexuais (item 22) $(\chi 2(1)$ $=3,939, p=0,047)$. Para os demais itens, não foi encontrada diferença em função da faixa etária. Dentre as meninas, o único item para o qual foi encontrada diferença entre as escolares e pré-escolares foi aquele que se refere a mostrar as partes sexuais para os adultos (item 20 ), comportamento mais frequentemente apontado por pais de meninas pré-escolares $(36,4 \%)$ do que de escolares $(20 \%)(\chi 2(1)=3,957, p=0,047)$.

A comparação entre os comportamentos reportados pelos responsáveis por meninos e meninas pré-escolares não indicou diferença significativa. Dentre os escolares, diferenças por sexo foram encontradas nos itens 9 (imita o ato sexual) $(\chi 2(1)=4,910, p=0,027)$ e 20 (mostra partes sexuais para adultos) $(\chi 2(1)=10,444, p$ $=0,001)$, ambos mais frequentes dentre os meninos.

Na soma total do questionário, os meninos apresentam, em média, mais comportamentos sexuais que as meninas $(8,04$ x 5,12, respectivamente). Essa diferença é estatisticamente significativa $(\mathrm{t}(1,52)=2,097 ; p=0,04)$. $\mathrm{Na}$ análise por faixa etária, não foi encontrada diferença estatística entre o que foi reportado pelos pais de pré-escolares e escolares $(\mathrm{t}(1,52)=0,374 ; p=0,710)$.

\section{2) Comparação qualitativa entre as crianças dos dois estudos (brasileiras e americanas)}

Os resultados encontrados no presente estudo foram comparados com os encontrados nos Estados Unidos $^{4}$. No Quadro 1 são apresentados os itens mais e menos assinalados nos dois estudos.

Quadro 1. Comparação qualitativa entre o estudo brasileiro e o norte-americano com relação aos comportamentos sexuais infantis mais frequentes

\begin{tabular}{|c|c|}
\hline $\begin{array}{c}\text { Estados Unidos - Friedrich e } \\
\text { col. (1991) }\end{array}$ & Brasil - Presente estudo \\
\hline \multicolumn{2}{|c|}{ Itens mais frequentes } \\
\hline $\begin{array}{l}35 \text {. Brinca com brinquedos do } \\
\text { sexo oposto }\end{array}$ & 11. Toca os genitais em casa \\
\hline $\begin{array}{l}\text { 5. Causa machucados nas coxas } \\
\text { e/ou ânus }\end{array}$ & 34. Interesse pelo sexo oposto \\
\hline 11. Toca os genitais em casa & $\begin{array}{l}\text { 35. Brinca com brinquedos do } \\
\text { sexo oposto }\end{array}$ \\
\hline $\begin{array}{l}24 \text {. Tira a roupa na frente dos } \\
\text { outros }\end{array}$ & $\begin{array}{l}\text { 6. Toca em seios (da mãe ou de } \\
\text { outra mulher) }\end{array}$ \\
\hline $\begin{array}{l}25 \text {. Senta-se expondo as coxas } \\
\text { ou roupas intimas }\end{array}$ & $\begin{array}{l}\text { 24. Tira a roupa na frente dos } \\
\text { outros }\end{array}$ \\
\hline \multicolumn{2}{|c|}{ Itens menos frequentes } \\
\hline $\begin{array}{l}\text { 10. Colocar boca em partes } \\
\text { sexuais }\end{array}$ & $\begin{array}{l}\text { 5. Causa machucados nas coxas } \\
\text { e/ou ânus }\end{array}$ \\
\hline $\begin{array}{l}\text { 15. Pede para participar de atos } \\
\text { sexuais }\end{array}$ & $\begin{array}{l}\text { 17. Insere objetos no ânus ou } \\
\text { vagina }\end{array}$ \\
\hline 7. Masturba-se com objetos & 7. Masturba-se com objetos \\
\hline $\begin{array}{l}\text { 17. Insere objetos no ânus ou } \\
\text { vagina }\end{array}$ & $\begin{array}{l}\text { 10. Colocar boca em partes } \\
\text { sexuais }\end{array}$ \\
\hline 9. Imita ato sexual & $\begin{array}{l}\text { 15. Pede para participar de atos } \\
\text { sexuais }\end{array}$ \\
\hline
\end{tabular}

Nota-se bastante semelhança na prevalência encontrada nos dois estudos. Dos cinco itens com comportamentos mais frequentemente encontrados na amostra brasileira, três aparecem também dentre os mais frequentes na amostra americana. Embora não apareçam nos dados aqui apresentados, deve-se notar que os itens 34 . "Parece muito interessado(a) no sexo oposto" e 6. "Toca ou tenta tocar os seios da mãe ou de outras mulheres", que estão entre os cinco mais frequentes no Brasil, estão 
entre os dez mais frequentes nos Estados Unidos. O item 25. "Senta expondo as coxas ou as roupas intimas", que está entre os mais frequentes da amostra americana, com $36,4 \%$ dos pais afirmando a ocorrência de tal comportamento nos últimos seis meses, aparece também na amostra brasileira em $24,1 \%$ dos casos.

Com relação aos itens que apresentam comportamentos sexuais infantis menos frequentes, quatro dos cinco itens são iguais nos dois estudos. Uma das diferenças envolve o item 9. "Imita o ato sexual", que apresenta porcentagem bem baixa nos Estados Unidos (1,1\%), mas é encontrado com frequência intermediária no Brasil (16,6\%). A maior discrepância entre os dois estudos está no item 5. "Causa machucados na região anal e nas coxas por excesso de manuseio", que não apareceu nesta amostra de crianças brasileiras e é o segundo mais frequente na amostra americana.

\section{3) Relação entre comportamentos sexuais e proble- mas de comportamento}

A relação entre comportamentos sexuais e problemas de comportamento foi analisada por meio das escalas de Internalização, Externalização e Total de Problemas de Comportamento do CBCL. Os escores médios obtidos pelas crianças da presente amostra estavam na faixa normal do instrumento. A relação entre comportamentos sexuais assinalados no CSBI e problemas de comportamentos avaliados por meio do CBCL foi analisada pela correlação bivariada de Pearson. Na Tabela 2 são apresentadas as correlações encontradas.

Tabela 2. Correlação entre o CSBI e as escalas de problemas de comportamento do CBCL

\begin{tabular}{l|c}
\hline & $\begin{array}{c}\text { Total de comportamentos } \\
\text { sexuais (CSBI) }\end{array}$ \\
\hline Escala de Internalização & $0,496^{*}$ \\
Escala de Externalização & $0,494^{*}$ \\
Escala Total & $0,549^{*}$ \\
\hline
\end{tabular}

Legenda: $* p<0,005$.

Foram encontradas correlações positivas moderadas entre o total de comportamentos sexuais apontados no CSBI e as escalas de problemas de comportamento avaliadas por meio do CBCL. Ou seja, no geral, os pais que apontaram mais comportamentos sexuais também apontaram mais problemas de comportamento em seus filhos, tanto para a escala de Internalização, quanto para a de Externalização e para a Total de Problemas de Comportamento.

\section{DISCUSSÃO}

O presente trabalho teve como objetivo principal avaliar a frequência de comportamentos sexuais observados por pais de crianças e estudar o efeito das variáveis idade e o sexo na presença e nos tipos de comportamentos apresentados. Para tanto, foi utilizado um instrumento internacionalmente reconhecido para avaliar comportamentos sexuais de crianças ${ }^{4,5,7}$ e adaptado para a realidade brasileira ${ }^{11}$. Os dados obtidos com a amostra do presente estudo sugerem que alguns comportamentos sexuais como "tocar as partes sexuais em casa", "ter interesse por brinquedos do sexo oposto", "brincar com brinquedos do sexo oposto", "tocar os seios da mãe ou de outra mulher" e "tirar a roupa na frente de outras pessoas", são bastante frequentes e comuns dentre meninos e meninas de quatro a oito anos.

Constata-se também que, apesar dos pais relatarem que as crianças apresentam várias respostas sexuais, tais como se interessar pelo sexo oposto, brincar com brinquedos do sexo oposto e despir-se na frente de outras pessoas, eles apontaram com baixa frequência, ou mesmo nem apontaram, respostas que envolviam a masturbação com objetos ou com outras pessoas e respostas envolvendo o ato sexual em si, seja ele com adultos ou mesmo outras crianças. Ou seja, as respostas sexuais são vistas pelos pais, porém as respostas que se referem diretamente ou fazem alusão ao ato sexual não são apresentadas no relato dos pais, exceto aquelas menos explícitas. Isso evidencia tanto a menor ocorrência de comportamentos mais explícitos, quanto à dificuldade metodológica de não se fazer uma observação direta dos comportamentos sexuais, que poderia fornecer dados mais precisos que o relato parental.

Assim como no trabalho de adaptação do CSBI para avaliação de crianças brasileiras ${ }^{11}$, os resultados do presente estudo sugerem que o uso do CSBI para ava- 
liar os comportamentos sexuais de crianças brasileiras é adequado, já que o padrão de frequência (de emissão e não emissão) de comportamentos específicos da maioria das crianças estudadas se assemelha bastante ao das americanas que participaram originalmente do estudo com o $\mathrm{CSBI}^{4}$. Embora a amostra do presente trabalho tenha sido muito pequena em comparação com a de Friedrich ${ }^{4}$, praticamente os mesmos comportamentos sexuais foram identificados como os mais frequentes, exceto pelo comportamento de causar machucados na região anal e nas coxas por excesso de manuseio, que é comum entre as crianças americanas e não apareceu no relato dos pais das crianças brasileiras estudadas. Em síntese, pode-se dizer que estas crianças não parecem ser, do ponto de vista do desenvolvimento sexual, diferentes das crianças americanas.

Ainda que poucas diferenças tenham sido encontradas em função do sexo, a média total obtida no CSBI foi maior dentre os meninos, o que é consistente com os dados da literatura internacional ${ }^{4,5,7}$. O fato de a genitália masculina estar mais exposta e disponível para manipulação pode ser uma explicação para os pais reportarem mais comportamentos sexuais em meninos. Além disso, fatores culturais e sociais devem ser considerados para entender esse resultado, visto que os valores sobre quais comportamentos são aceitáveis para meninos e meninas geralmente são diferentes e isso pode afetar tanto a ocorrência, quanto a frequência com que os comportamentos são relatados ${ }^{12}$.

$O$ fato de os comportamentos sexuais serem apontados pela literatura internacional como mais frequentes dentre as crianças mais novas tem sido explicado em função do desenvolvimento cognitivo da criança, que conforme vão crescendo, possivelmente ficam muito mais sob controle de aspectos morais relativos ao sexo impostos pela cultura ${ }^{4,5,7}$. No presente estudo, não foram encontradas diferenças na pontuação média total das crianças escolares e pré-escolares. Diferenças pontuais em itens, no entanto, foram encontradas, especialmente dentre os meninos, sendo que foi indicada com maior frequência o uso de palavras sexuais, o olhar fotos de pessoas nuas e a fala sobre atos sexuais. Nota-se, entretanto, que os itens para os quais foi encontrada diferença exigem um repertório mais elaborado e acesos a conteú- dos que uma criança pré-escolar sem histórico de abuso sexual raramente teria acesso. Dentre as meninas, foi encontrada diferença na frequência em que mostram as partes sexuais para adultos, maior dentre as pré-escolares. Essa diferença pode ser compreendida como fruto da moralidade sexual feminina que é ensinada pela sociedade conforme a criança vai crescendo, pela percepção ambivalente da mulher como protetora da moralidade e símbolo sexual ${ }^{13,14}$.

Além de verificar a frequência dos comportamentos sexuais infantis, o presente estudo se propôs a verificar se estes estavam relacionados com problemas de comportamento, como vêm sendo reportado em outros estudos da área ${ }^{1,3 \cdot 7}$. As correlações positivas moderadas encontradas no presente estudo entre os comportamentos sexuais e problemas de comportamento Internalizantes, Externalizantes e Totais indicam que os pais que reportam mais comportamentos sexuais também indicam a presença de mais problemas de comportamento em seus filhos. Esse resultado sugere que as crianças que manifestam mais problemas emocionais e comportamentais tendem a apresentar com mais frequência diversos tipos de comportamentos, inclusive os sexuais?.

Tanto o CBCL quanto o CSBI se mostraram como instrumentos bastante úteis para a prática do profissional de saúde infantil, pois ambos servem como base para o início de um diagnóstico ou avaliação mais apurada. O CBCL apresenta escalas que envolvem diversas classes de comportamentos, o que facilita a avaliação ou triagem. O CSBI, por sua vez, é um facilitador para o estabelecimento de um diálogo com os pais da criança sobre sexualidade, visto que cria a possibilidade dos cuidadores exporem suas opiniões sobre o tema de maneira não constrangedora, visto que se trata de um assunto pouco discutido e considerado ainda como tabu na sociedade.

Os resultados apontam que os comportamentos sexuais, comumente apresentados durante o desenvolvimento infantil, estão presentes na amostra estudada. Além disso, eles apontam quais desses comportamentos são mais frequentes e quais deles merecem atenção dos pais e profissionais que trabalham com criança, em função de sua raridade. Entretanto, isso não significa que as crianças saibam como se comportar sexualmente, ou que já sabem tudo sobre o assunto e, portanto, nada lhes é 
preciso ensinar, ou que o conhecimento sobre sexualidade seria um perigo estimulando-as a terem atividades sexuais precocemente, como preconizam os mitos apontados na literatura ${ }^{6}$. Pelo contrário, justamente porque os comportamentos sexuais ocorrem, especialmente em crianças bem pequenas, é que as mesmas precisam ser orientadas sobre sua sexualidade para que elas fiquem em uma situação de menos risco para experiências adversas que podem impactar negativamente seu desenvolvimento e para evitar que comportamentos mal adaptados sejam desenvolvidos ${ }^{6,15,17}$.

Pais podem evitar falar com seus filhos sobre sexo por diversos fatores, incluindo a falta de consciência da necessidade, falta de informação sobre o tema, vergonha, baixa habilidade para conversar sobre o assunto, falta de confiança e falta de habilidades de comunicação. Entretanto, iniciar a educação sexual enquanto as crianças são bem novas traz múltiplos benefícios para a criança e para os pais, já que os pais entendem mais fácil falar sobre o assunto nessa idade e, dessa forma, conseguem construir uma boa base para diálogos, e isso reduz o risco de gravidez na adolescência ${ }^{3,15,16}$.

Os pais e a sociedade tem papel muito significativo na educação e prevenção de distúrbios sexuais e de problemas de saúde relacionados ao sexo ${ }^{17}$, já que são eles os responsáveis por promover um ambiente saudável de interação, que incentive relações interpessoais mais íntimas entre pais e filhos, e devem colocar assuntos referentes à sexualidade de forma natural e espontânea. Dessa forma, conhecer os comportamentos sexuais mais comumente apresentados pelas crianças de uma determinada cultura permite com que os pais e os profissionais que lidam com crianças identifiquem, com mais facilidade, problemas nesta área ao longo do desenvolvimento.

Espera-se que as informações derivadas desse estudo, consistentes com diversos trabalhos realizados em outros países ${ }^{4,7}$, possam ser usadas por profissionais que trabalham com crianças para ajudar os pais a identificarem a relativa normalidade de um grande número de comportamentos sexuais. Considerando a relação entre comportamentos sexuais e abuso sexual relatada na literatura ${ }^{9}$, é importante que os profissionais tenham informações suficientes para orientar pais e crianças sobre o desenvolvimento sexual saudável.

Ao mesmo tempo em que é imprescindível que sejam obtidos dados sobre comportamentos sexuais na infância, é clara a dificuldade em identificar os comportamentos realmente comuns, pois os dados são sempre colhidos de forma indireta (entrevistas com os pais ou professores) ou por meio de entrevistas retrospectivas com adultos a respeito de sua infância. O uso de entrevista com os pais para avaliar os comportamentos sexuais de crianças deve ser discutido. Sugere-se que a observação direta sistemática e a resposta dos pais a entrevistas são os métodos recomendáveis. No entanto, depois da primeira infância, os pais progressivamente tornam-se menos úteis como informantes, pois sabem menos sobre os pensamentos e atividades sexuais de seus filhos ${ }^{2}$. Muitas vezes os pais ficam bem pouco tempo com os filhos e têm pouca oportunidade de presenciar seus comportamentos sexuais. Além disso, é bem provável também que a criança dissimule ou esconda seus comportamentos sexuais, pois na cultura ocidental muitos dos comportamentos sexuais que seriam naturais na infância são reprimidos pelos adultos. $\mathrm{O}$ fato de seis participantes terem preenchido o inventário CSBI com a opção 0 (nunca) para todos os itens, indicando que nos últimos seis meses aquelas crianças não apresentaram nenhum dos 35 comportamentos listados, pode ilustrar o tipo de dificuldade mencionada acima. Dessa forma, é importante levar em consideração o fator informante na análise dos dados obtidos sobre os comportamentos sexuais infantis ${ }^{3}$.

Além dos dados terem sido obtidos apenas com os responsáveis pelas crianças, outras limitações estão presentes neste estudo. Trata-se de um estudo transversal, com amostra pequena e de conveniência, que utilizou o relato dos pais para verificar o histórico de abuso sexual. Estudos posteriores podem investir na avaliação do contexto familiar da criança, além de incluir amostras mais amplas e com crianças que sofreram abuso sexual e que apresentam distúrbios de ordem sexual, como no trabalho de Friedrich et al. ${ }^{9}$, para que os dados possam ser contrastados ao de uma população tida como normal e compreender melhor tanto quais são os comportamentos sexuais emitidos pelas crianças durante as diferentes fases do desenvolvimento e quais devem servir de alerta para questões associadas com o abuso sexual. 


\section{CONCLUSÃO}

$\mathrm{O}$ presente estudo evidenciou a presença de comportamentos sexuais nas crianças brasileiras de maneira semelhante à anteriormente apontada em estudos internacionais no que se refere a tipos de comportamentos emitidos e frequência observada pelos pais. Além disso, o mesmo padrão de diferença por sexo foi registrado, com os meninos apresentando mais comportamentos sexuais que as meninas. Os pais que relataram mais comportamentos sexuais reportaram também mais problemas de comportamento, confirmando a associação entre essas duas variáveis.

\section{REFERÊNCIAS}

1. Kellogg N. The Committee on Child Abuse and Neglect. Clinical Report - The evaluation of sexual behaviors in children. Pediatr. 2009;124(3):992-8. doi: 10.1542/peds.2009-1692

2. Meyer-Bahlburg HFL, Steel JL. Using the parents as a source of information about the child: With special emphasis on the sex problems scales of the Child Behavior Checklist. In: Bancroft J, organizador. Sexuality Development in Childhood. Indiana University Press: Bloomington; 2003. p. 34-46.

3. Allen B. Children with sexual behavior problems: Clinical characteristics and relationship to child maltreatment. Child Psychiatry Hum Dev. 2017;48(2):189-99. doi:10.1007/s10578-016-0633-8

4. Friedrich WN, Grambsch P, Broughton D, Kuiper J, Beike RL. Normative sexual behavior in children. Pediatr. 1991;88(3):456-64.

5. Friedrich WN, Fischer JL, Broughton D, Houston M, Constance RS. Normative sexual behavior in children: A contemporary study. Pediatr. 1998;101(4):e9. doi: 10.1542/peds.101.4.e9

6. Gordon BN, Schroeder CS. Sexuality: a developmental approach to problems. New York: Plenum Press; 1995.
7. Schoentjes E, Deboutte D, Friedrich WN. Child Sexual Behavior Inventory: A Dutch-speaking normative sample. Pedriatr. 1999;104(4):885-93.

8. Achenbach TM. Integrative guide for the $\mathrm{CBCL} / 4-18$, YSR, and TRF profiles. Burlington, Vermont: University of Vermont; 1991.

9. Friedrich WN, Fischer JL, Dittner CA, Acton R, Berliner L, Butler J, et al. Child Sexual Behavior Inventory: Normative, Psychiatric, and Sexual Abused Comparisons. Child Maltreatment. 2001;6(1):37-49. doi: $10.1177 / 1077559501006001004$

10. Bordin IA, Rocha MM, Paula CS, Teixeira MCTV., Achenbach Thomas M., Rescorla Leslie A. et al . Child Behavior Checklist (CBCL),Youth Self-Report (YSR) and Teacher's Report Form(TRF): an overview of the development of the original and Brazilian versions. Cad. Saúde Pública [Internet]. 2013;29(1):13-28. Disponível em: doi: 10.1590/S0102-311X2013000100004.

11. Rossetti MO, Assumpção-Junior FB. Inventário de comportamentos sexuais da criança: adaptação brasileira e análise de evidências de validade. Bol. Acad. Paulista de Psicologia. 2014;34(86):208-31.

12.Josephs L. How Children Learn About Sex: A cross -species and cross-cultural analysis. Arch Sex Behav. 2015;44(4):1059-69. Disponível em: doi: 10.1007/ s10508-015-0498-0

13. Chauí M. Participando do debate sobre mulher e violência. In: Várias Autoras. Perspectivas Antropológicas da Mulher, $n^{\circ}$ 4, Rio de Janeiro: Zahar; 1984. p. 23-62.

14.Goddard V. Honour and shame: the control of women's sexuality and group identity in Naples. In: Caplan P, editor. The Cultural Construction of Sexuality. London/New York: Routledge; 1987. p. 166-92.

15. Silvares EFM. Orientação sexual da criança. In: Brandão MZS, Conte FC, Mezzaroba SMB, organizadoras. Comportamento Humano: tudo (ou quase tudo) que você gostaria de saber para viver melhor. Santo André: ESETec; 2002. p. 111-20. 
16. Silvares EFM. Sexualidade na infância: Quando e como intervir. In: Marinho ML, Caballo VE, organizadores. Psicologia clínica e da saúde. Londrina: Editora da Universidade Estadual de Londrina; 2001. p. 77-93.

17. Kenny MC, Wurtele SK. Latino Parents' Plans to Communicate About Sexuality With their Children. Journal of Health Communication. 2013;18(8):931-42. Disponível em: doi: 10.1080/10810730.2012.757397.

Recebido em: 29/07/2017 Aceito em: 01/03/2018 